\title{
Safety and efficacy evaluation of low-dose trimethoprim-sulfamethoxazole for prophylaxis of Pneumocystis pneumonia in HIV uninfected patients undergoing hemodialysis: a retrospective observational study
}

Kanae Yamashita ${ }^{1 *}$, Yoshimitsu Shimomura², Hiroaki Ikesue ${ }^{1}$, Nobuyuki Muroi ${ }^{1}$, Akihiro Yoshimoto ${ }^{3}$ and Tohru Hashida ${ }^{1}$

\begin{abstract}
Background: Pneumocystis pneumonia (PCP) is a potentially life-threatening infection. Trimethoprimsulfamethoxazole (TMP-SMX) is considered as the first regimen for PCP prophylaxis according to several guidelines. The recommended prophylactic dose of TMP-SMX has been determined based on patients with normal renal function, but the appropriate dosage for patients undergoing hemodialysis is unknown. The aim of this study was to investigate the efficacy and safety of low-dose TMP-SMX in patients undergoing hemodialysis.

Methods: HIV-uninfected adult patients who were undergoing hemodialysis and administered TMP-SMX for PCP prophylaxis, were included, and divided into standard-dose ( $\geq 6$ single strength (SS, TMP-SMX $80 \mathrm{mg} / 400 \mathrm{mg}$ tablets/week) and low-dose groups (<6 SS tablets/week). The endpoints were cumulative incidence of PCP and cumulative discontinuation rate of TMP-SMX due to adverse events. For comparison of the groups, we employed the chi-squared test for categorical variables and the Mann-Whitney $U$ test for continuous variables. Risk factors for the endpoints were evaluated using the Cox Fine and Gray method.
\end{abstract}

Results: The median age of the 81 patients included in the study was 67 years (IQR: 60-76 years), and 52 patients (64.2\%) were men. No patients in either group developed PCP during the observation period. The yearly cumulative incidence of discontinuation was 12.1\% (95\% confidence interval [Cl]: 0.027-0.29) in the low-dose group and 35.6\% (95\% Cl: $0.20-0.52)$ in the standard-dose group $(P=0.019)$. The adjusted hazard ratio of the low-dose group compared to standard-dose group was 0.18 ( $95 \%$ Cl: $0.04-0.86, P=0.032$ ).

\footnotetext{
* Correspondence: yamashitakanae@kcho.jp

'Department of Pharmacy, Kobe City Medical Center General Hospital, 2-1-1

Minatojima-minamimachi, Chuo-ku, Kobe, Hyogo 650-0047, Japan

Full list of author information is available at the end of the article
}

C C The Author(s). 2021 Open Access This article is licensed under a Creative Commons Attribution 4.0 International License, which permits use, sharing, adaptation, distribution and reproduction in any medium or format, as long as you give appropriate credit to the original author(s) and the source, provide a link to the Creative Commons licence, and indicate if changes were made. The images or other third party material in this article are included in the article's Creative Commons licence, unless indicated otherwise in a credit line to the material. If material is not included in the article's Creative Commons licence and your intended use is not permitted by statutory regulation or exceeds the permitted use, you will need to obtain permission directly from the copyright holder. To view a copy of this licence, visit http://creativecommons.org/licenses/by/4.0/ The Creative Commons Public Domain Dedication waiver (http://creativecommons.org/publicdomain/zero/1.0/) applies to the data made available in this article, unless otherwise stated in a credit line to the data. 
Conclusions: None of the study patients developed PCP, and the cumulative discontinuation rate of TMP-SMX due to adverse events was significantly lower in the low-dose group compared to that in the standard-dose group $(P=$ 0.032). These results indicate that low-dose TMP-SMX is an appropriate regimen to maintain a balance between PCP prophylaxis and prevention of adverse events due to TMP-SMX administration. These findings can guide health care professionals to determine TMP-SMX dosage when considering PCP prophylaxis for patients undergoing hemodialysis.

Keywords: Trimethoprim-sulfamethoxazole, Pneumocystis pneumonia, Prophylaxis, Hemodialysis, Low-dose

\section{Background}

Pneumocystis pneumonia (PCP) is a potentially lifethreatening infection that occurs in both patients with human immunodeficiency virus (HIV) infection and those who are immunocompromised without HIV infection (HIV-uninfected) [1]. The mortality rate of PCP in immunocompromised HIV-uninfected patients is especially high, at $35-50 \%[2-5]$. Therefore, certain guidelines recommend the use of $\mathrm{PCP}$ prophylaxis in immunocompromised HIV-uninfected patients $[1,6,7]$. Trimethoprim-sulfamethoxazole (TMP-SMX) has been considered the first-line prophylactic regimen for PCP according to several guidelines $[1,6,8,9]$.

The standard dosage of TMP-SMX for PCP prophylaxis is TMP-SMX $80 \mathrm{mg} / 400 \mathrm{mg}$ or TMP-SMX $160 \mathrm{mg} /$ $800 \mathrm{mg}$, either daily or three times a week, i.e., 6-14 single strength (SS) tablets/week is considered the standard dosage [1]. Additionally, trimethoprim and sulfamethoxazole are excreted renally; therefore, patients with impaired renal function exhibit an increase in the half-lives of both components, requiring dosage adjustment of the drugs based on creatinine clearance $[1,9]$. Moreover, the recommended prophylactic dose of TMP-SMX has been determined based on studies in patients with normal renal function, and little evidence is available regarding the appropriate prophylactic dosage of TMP-SMX against PCP in immunocompromised HIV-uninfected patients undergoing hemodialysis. Therefore, the aim of this study was to investigate the efficacy and safety of low-dose TMP-SMX in patients undergoing hemodialysis.

\section{Methods}

This single center, retrospective, observational study included 116 HIV-uninfected adult patients who had undergone hemodialysis at Kobe City Medical Center General Hospital between January 1, 2012 and December 31, 2017, and were administered TMP-SMX for prophylaxis against PCP. Among them, we excluded patients who were under a short observation period of less than 14 days $(n=35)$. The patients were divided into two groups, standard-dose group and low-dose group, according to the TMP-SMX dose administered. The standard dose was defined as $\geq 6$ SS tablets/week and low-dose was defined as < 6 SS tablets/week [1, 10]. With regards to underlying diseases, leukemia, lymphoma, and multiple myeloma were collectively defined as hematological malignancies. Nephrosis, polyangiitis, connective tissue disease, and idiopathic thrombocytopenic purpura (ITP) were collectively defined as autoimmune diseases. Corticosteroid doses were expressed in Prednisolone (PSL) equivalents. If the doses of corticosteroids were tapered during the observation period, the initial dose was defined as the steroid dose. Further, if steroids were administered only on certain days during a period of time, such as when chemotherapy and corticosteroid were used together, the average daily dose during the period was defined as the corticosteroid dose.

The severity of adverse events (AEs) was determined using the Common Terminology Criteria for Adverse Events (CTCAE), version 5.0 [11]. The study was conducted in accordance with the principles of the Declaration of Helsinki. This study was approved by the ethics committee in Kobe City Medical Center General Hospital, and the reference number was zn190616. Informed Consent was waived by the ethics committee in Kobe City Medical Center General Hospital because this study used retrospective data obtained from hospital records and there were no interventions in the study patients.

The cumulative incidence of PCP and cumulative discontinuation rate of TMP-SMX due to AEs were set as endpoints; death was considered as a competing risk.

Categorical variables were summarized using numbers and percentages, and continuous variables were summarized using medians (interquartile range [IQR]). For comparison of the groups, we employed the chi-squared exact test for categorical variables and the MannWhitney $U$ test for continuous variables.

The observation period for this analysis was fixed at June 30, 2019. Event rates were estimated with a 95\% confidence interval (CI) for the endpoints. Gray's method was employed to consider the competing risks. We evaluated risk factors for endpoints using the Cox Fine and Gray method, which were described as adjusted hazard ratio (aHR) and 95\% CI. The Fine and Gray method was employed to consider competing risks. The adjusted covariates were selected clinically and included the following: age, sex, dosage of TMP-SMX, underlying 
disease, and treatment. The threshold for statistical significance was set at $P<0.05$.

All statistical analyses were performed with EZR (Saitama Medical Center, Jichi Medical University, Saitama, Japan), which is a graphical user interface for $\mathrm{R}$ [12].

\section{Results}

A total of 81 patients were included in the study as follows: 36 patients in the low-dose group and 45 patients in the standard-dose group. The median age was 67 years (IQR: $60-76$ years), and 52 patients (64.2\%) were men. Regarding underlying diseases, 23 patients had hematological malignancies, 45 had autoimmune diseases, and 13 had other diseases. Among the patients with hematological malignancies, five had leukemia, nine had lymphoma, and nine had multiple myeloma. Among those with autoimmune diseases, 10 had connective tissue disease, 17 had polyangiitis, 17 had nephropathy, and one had ITP. Among those with other diseases, six had pulmonary disease, three had eosinophilia, and one patient each had drug eruption, adrenocortical insufficiency, encephaloma, and aldosteronism. Most patients with hematological malignancies received chemotherapy (95.6\%); 14 patients among them were treated with a regimen of chemotherapy including corticosteroids. Furthermore, most patients (97.8\%) with autoimmune disease received corticosteroids; 30 patients among them received steroids alone while 14 patients received a combination of steroids and immunosuppressive drugs. The median dose of corticosteroid was $30 \mathrm{mg}$ (IQR: 20-40 $\mathrm{mg}$ ). The baseline characteristics of the patients and the breakdown of immunosuppressive treatments administered to each patient are described in Table 1. There were no significant differences in age, sex, weight distribution, or treatment between the two groups. Particularly, the low-dose group included more patients with hematological malignancies and fewer patients with autoimmune disease than the standard-dose group.

The median observation period of patients without events was 187 days (IQR: 60-458 days) in the low-dose group and 201 (IQR: 49-459 days) in the standard-dose group $(P=0.586)$. None of the patients in either group developed PCP during the observation period. The cumulative incidence of discontinuation was $12.1 \%(95 \%$ CI: $0.027-0.29)$ in the low-dose group and $35.6 \%$ (95\% CI: $0.20-0.52)$ in the standard group ( $P=0.019$, Fig. 1$)$. The aHR of the low-dose group compared to that of the standard-dose group was 0.18 (95\% CI: $0.04-0.86, P=$ 0.032) (Table 2).

The most frequent AE that caused TMP-SMX discontinuation in the low-dose group was thrombocytopenia and leukocytopenia, while in the standard-dose group, it was rash, thrombocytopenia, anemia, and hyponatremia (Table 3).

\section{Discussion}

In the present study, we retrospectively compared the efficacy and safety of low-dose and standard-dose TMPSMX for prophylaxis against PCP in 81 immunocompromised HIV-uninfected patients undergoing hemodialysis. With regards to efficacy, no patients developed PCP in both the low-dose and standard-dose groups. Additionally, the discontinuation rate of TMPSMX regimen among the low-dose group was lower than that in the standard-dose group, suggesting that a low-dose of TMP-SMX may be better tolerated than standard-dose in patients undergoing hemodialysis.

Several studies have compared the therapeutic and prophylactic effects of low-dose and standard dose of TMP-SMX. Prophylaxis of PCP with 6-14 SS tablets/ week of TMP-SMX, was associated with a similar development rate of PCP in several reports. Therefore, the guidelines recommend 6-14 SS tablets/week of TMPSMX for prophylaxis of PCP. In addition, low-dose TMP-SMX was administered to patients with normal renal function. For example, an open-label randomized control study of patients with rheumatoid arthritis revealed that the development of PCP was similar between the standard-dose group (7 SS/week) and low-dose group (3.5 SS/week) [13]. Another retrospective study of patients who underwent allogeneic hematopoietic stem cell transplantation revealed a similar efficacy between low-dose (4 SS/week) and standard-dose TMP-SMX (7 SS/week) in 156 patients [13, 14]. These studies included a few patients with creatinine clearance of $<30 \mathrm{~mL} / \mathrm{min}$; however, no patients were undergoing hemodialysis. The prophylactic efficacy of TMP-SMX was expected to be similar or higher in patients with impaired renal function than in patients with normal renal function because the drugs were renally excreted and half-lives of both components increased in patients with impaired renal function. As expected, our study revealed that the development of PCP was not observed in either the low-dose (5 SS/week) or standard-dose (6-14 SS/week) TMPSMX groups. These results suggest that low-dose TMPSMX administration may be an option for patients undergoing hemodialysis.

Serious AEs have often resulted in the discontinuation of TMP-SMX regimen, and several trials have been conducted to explore safer regimens that will allow continuation of treatment or prophylaxis. A Cochrane review of four trials comparing TMP-SMX prophylaxis (6-14 SS/ week) versus placebo or no intervention showed no significant difference in the rate of AEs between the two groups, suggesting the safety of prophylactic TMP-SMX in patients with normal renal function [15]. Moreover, some studies investigating a lower dose of TMP-SMX for prophylaxis of PCP reported that the low-dose TMPSMX (6 SS/week) group had a lower discontinuation 
Table 1 Baseline characteristics of patients in the low-dose and standard-dose groups

\begin{tabular}{|c|c|c|c|}
\hline Characteristics & $\begin{array}{l}\text { Low-dose } \\
(n=36)\end{array}$ & $\begin{array}{l}\text { Standard-dose } \\
(n=45)\end{array}$ & $P$-value \\
\hline Age (years), median (IQR) & $67(59-76)$ & $67(60-76)$ & 0.850 \\
\hline Male sex, n (\%) & $25(69.4)$ & $27(60.0)$ & 0.490 \\
\hline Weight (kg), median (IQR) & $57.4(50.0-64.8)$ & $56.1(50.0-63.9)$ & 0.530 \\
\hline Underlying disease, $\mathrm{n}(\%)$ & & & $<0.001$ \\
\hline Hematological malignancy, n (\%) & $18(50.0)$ & $5(11.1)$ & \\
\hline Leukemia, n (\%) & $5(13.9)$ & $0(0.0)$ & \\
\hline Lymphoma, n (\%) & $6(16.7)$ & $3(6.7)$ & \\
\hline Multiple myeloma, n (\%) & $7(19.4)$ & $2(4.4)$ & \\
\hline Autoimmune disease, n (\%) & $12(33.3)$ & $33(73.3)$ & \\
\hline Connective tissue disease, n (\%) & $6(16.7)$ & $4(8.9)$ & \\
\hline Polyangiitis, n (\%) & $3(8.3)$ & $14(31.1)$ & \\
\hline Nephritis, n (\%) & $2(5.6)$ & $15(33.3)$ & \\
\hline ITP, n (\%) & $1(2.8)$ & $0(0.0)$ & \\
\hline Others ${ }^{\mathrm{a}}, \mathrm{n}(\%)$ & $6(16.7)$ & $7(15.6)$ & \\
\hline Treatment for each underlying disease & & & $<0.001$ \\
\hline \multicolumn{4}{|l|}{ Hematological malignancy } \\
\hline Chemotherapy, n (\%) & $6(16.7)$ & $2(4.4)$ & \\
\hline Chemotherapy with corticosteroid, $\mathrm{n}(\%)$ & $11(30.5)$ & $3(6.7)$ & \\
\hline Immunosuppressive agent, n (\%) & $1(2.8)$ & $0(0.0)$ & \\
\hline \multicolumn{4}{|l|}{ Autoimmune disease, n (\%) } \\
\hline Corticosteroid, n (\%) & $11(30.6)$ & $33(73.3)$ & \\
\hline Immune suppressive drug, $\mathrm{n}(\%)$ & $1(2.8)$ & $0(0.0)$ & \\
\hline \multicolumn{4}{|l|}{ Others } \\
\hline Corticosteroid, n (\%) & $5(13.9)$ & $7(15.6)$ & \\
\hline Chemotherapy, n (\%) & $1(2.8)$ & $0(0.0)$ & \\
\hline Dose of corticosteroid $(\mathrm{mg})^{\mathrm{b}}$, median (IQR) & $25.0(16.6-40.0)$ & $35(25.0-44.5)$ & 0.212 \\
\hline Dose of corticosteroid in patients with Hematological malignancy & $23.8(18.6-38.0)$ & $24.0(23.9-31.0)$ & \\
\hline Dose of corticosteroid in patients with autoimmune disease & $25.0(20.0-45.0)$ & $35.0(25.0-45.0)$ & \\
\hline Dose of corticosteroid in patients with other disease & $40.0(13.0-50.0)$ & $30.0(20.0-47.0)$ & \\
\hline Total bilirubin (mg/dL), median (IQR) & $0.4(0.3-0.5)$ & $0.4(0.3-0.6)$ & 0.626 \\
\hline ALT (IU/L), median (IQR) & $16.5(8.0-42.5)$ & $13.0(9.0-26.0)$ & 0.631 \\
\hline AST (IU/L), median (IQR) & $14.8(7.0-35.3)$ & $14.8(13.0-24.0)$ & 0.143 \\
\hline White blood cell $\left(\times 10^{3} / \mu \mathrm{L}\right)$, median (IQR) & $7.5(5.2-11.8)$ & $8.5(6.7-11.2)$ & 0.206 \\
\hline Hemoglobin (g/dL), median (IQR) & $9.65(8.8-11.3)$ & $9.9(9.0-11.6)$ & 0.450 \\
\hline Platelet $\left(\times 10^{4} / \mu \mathrm{L}\right)$, median $(\mathrm{IQR})$ & $15.7(11.8-25.4)$ & $18.7(14.8-25.6)$ & 0.150 \\
\hline
\end{tabular}

Categorical variables were summarized as counts and percentages, and continuous variables were summarized as medians and interquartile ranges (quartiles $1-$ 3). $P$ values were determined using the chi-squared exact test for categorical variables and the Mann-Whitney $U$ test for continuous variables Abbreviations: IQR interquartile range, ITP idiopathic thrombocytopenic purpura, ALT alanine aminotransferase, AST aspartate aminotransferase a Others included eosinophilia, Lung disease, drug eruption, adrenal cortex insufficiency, encephaloma and aldosteronism

${ }^{b}$ Corticosteroid doses are expressed in Prednisolone (PSL) equivalents

rate than the standard-dose TMP-SMX (7-14 SS/week) group. For example, the discontinuation rates attributable to adverse events were significantly lower in the half-strength group (19.1\%) than in the single strength group (41.8\%) in patients with rheumatoid arthritis [16].
Another study revealed that the discontinuation rate with low-dose TMP-SMX prophylaxis due to adverse events was low $(1.3 \%)$ in patients after allogeneic hematopoietic stem cell transplantation [13]. In addition, yet another study revealed that the discontinuation rate 


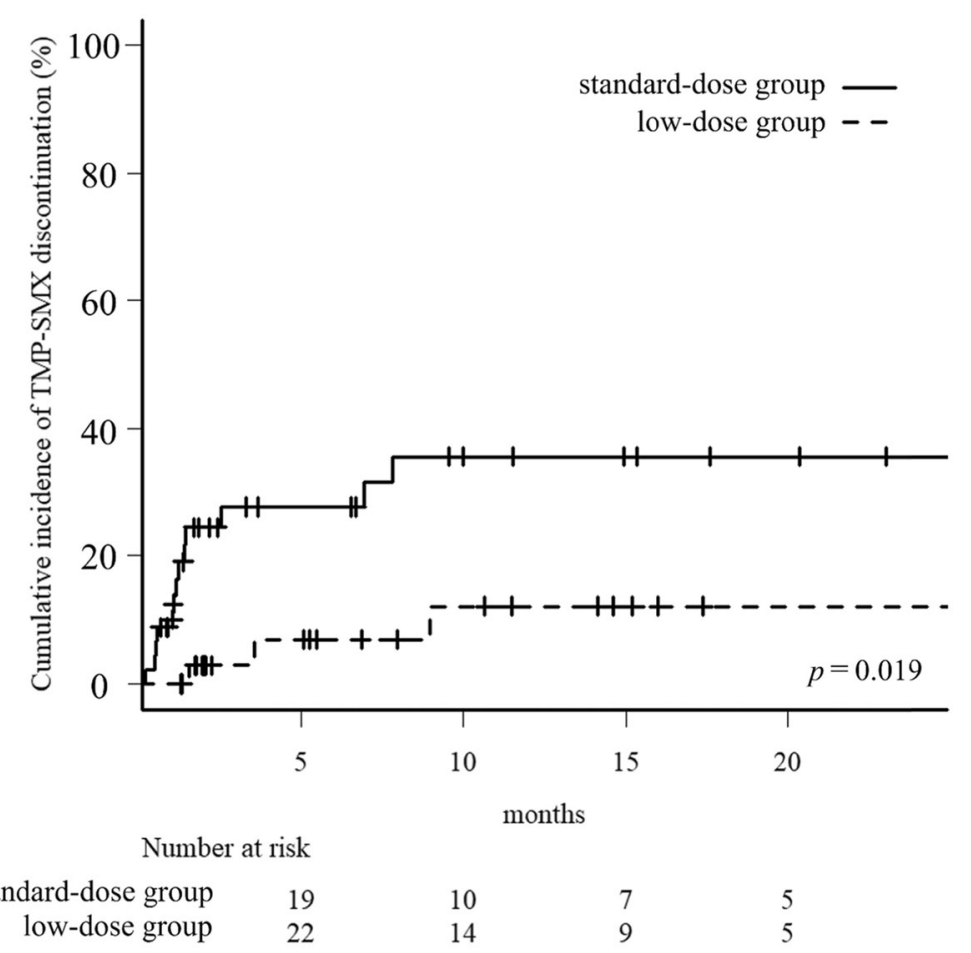

Fig. 1 Kaplan-Meier curves for comparison of time to drug discontinuation in patients undergoing hemodialysis. Aplan-Meier curves for comparison of time to drug discontinuation in patients undergoing hemodialysis, who were administered sulfamethoxazole-trimethoprim for the prophylaxis of Pneumocystis pneumonia. Abbreviations: TMP-SMX, trimethoprim-sulfamethoxazole

with a single strength TMP-SMX tablet three times a week for a year, due to adverse events was $10 \%$ in kidney transplant recipients [17]. Similar to these studies, our results showed that the cumulative discontinuation rate was lower in the low-dose group (12\%) than that in the

Table 2 Factor for TMP-SMX discontinuation rate

\begin{tabular}{llll}
\hline Factor & HR & $\mathbf{9 5 \% ~ C l}$ & $\boldsymbol{P}$-value \\
\hline Low-dose group (vs standard-dose) & 0.18 & $0.04-0.86$ & 0.032 \\
Age (over 60 years) & 2.54 & $0.56-11.5$ & 0.230 \\
Sex (men vs female) & 1.61 & $0.56-4.7$ & 0.380 \\
Disease & & & \\
$\quad$ Hematological malignancy (reference) & 1.00 & - & - \\
$\quad$ Autoimmune disease & 1.37 & $0.29-6.4$ & 0.690 \\
$\quad$ Other disease & 0.26 & $0.02-3.4$ & 0.310 \\
Treatment & & & \\
$\quad$ Low PSL dose & 1.00 & & \\
$\quad$ High PSL dose & 0.30 & $0.05-1.9$ & 0.200 \\
$\quad$ Others & 0 & $0-0$ & $<0.001$ \\
\hline
\end{tabular}

The risk factors for TMP-SMX discontinuation using the Cox Fine and Gray method were described as adjusted hazard ratios and $95 \% \mathrm{Cls}$. The adjusted covariates were selected clinically and included the following: age, sex, dosage of TMP-SMX, and underlying disease

Abbreviations: TMP-SMX trimethoprim-sulfamethoxazole, $H R$ hazard ratio, $\mathrm{Cl}$ confidence interval, PSL Prednisolone standard-dose group (36\%). Our results suggest that by adjusting the dosage of TMP-SMX to $<6$ SS tablets/ week, patients undergoing hemodialysis may be able to continue with the regimen as safely as patients with normal renal function. According to the above results, we considered that low-dose TMP-SMX is an appropriate dose that could maintain a balance between the prophylactic effects for PCP and therapeutic safety.

Our study has several limitations. First, this was a single-center, retrospective, observational study and our data were insufficient to eliminate confounding bias. Second, the incidence of PCP is rare in this population; therefore, the results of this study might lack statistical power for detecting the development of PCP. However, in terms of safety, we found that most of the treatment interruptions due to adverse events occurred early, within the first 12 months after initiation. Hence, the results of this study suggest that a lower dose regimen is expected to be safer compared to a higher dose regimen. Third, we could not perform drug concentration monitoring post hemodialysis, would have more accurately reflected the effects of hemodialysis. We believe that prospective studies that incorporate drug monitoring are needed in the future. However, data on prophylactic doses of TMP-SMX in patients undergoing hemodialysis are limited; therefore, we believe this study is meaningful 
Table 3 Adverse event requiring TMP-SMX discontinuation

\begin{tabular}{lll}
\hline Adverse event & $\begin{array}{l}\text { Low-dose } \\
\text { group }(\boldsymbol{n}=\mathbf{3 6})\end{array}$ & $\begin{array}{c}\text { Standard-dose } \\
\text { group ( } \mathbf{n = 4 5 )}\end{array}$ \\
\hline Number of discontinued patients, $\mathrm{n}(\%)$ & $3(8.3)$ & $13(28.9)$ \\
Details of adverse events & & $5(11.1)$ \\
Fever, $\mathrm{n}(\%)$ & $0(0.0)$ & $6(13.3)$ \\
Rash, $\mathrm{n}(\%)$ & $0(0.0)$ & $2(4.4)$ \\
Anorexia, $\mathrm{n}(\%)$ & $0(0.0)$ & $6(13.3)$ \\
Thrombocytopenia, $\mathrm{n}(\%)$ & $3(8.3)$ & $2(4.4)$ \\
Leukocytopenia, $\mathrm{n}(\%)$ & $3(8.3)$ & $6(13.3)$ \\
Anemia, $\mathrm{n}(\%)$ & $2(5.6)$ & $2(4.5)$ \\
Hyperkalemia, $\mathrm{n}(\%)$ & $1(2.8)$ & $6(13.3)$ \\
Hyponatremia, $\mathrm{n}(\%)$ & $2(5.6)$ & $5(11.1)$ \\
Increased alanine aminotransferase, $\mathrm{n}(\%)$ & $0(0.0)$ & $5(11.1)$ \\
Increased aspartate aminotransferase, $\mathrm{n}(\%)$ & $0(0.0)$ & \\
\hline
\end{tabular}

The number of patients who discontinued due to adverse events and details of adverse events reported in each group are shown. Thirteen patients in the standard-dose group and three patients in the low-dose group discontinued TMP-SMX due to adverse events. Some patients experienced multiple adverse events Abbreviation: TMP-SMX trimethoprim-sulfamethoxazole

in demonstrating that low-dose regimens may be safely continued.

\section{Conclusions}

PCP did not develop in either the low-dose or standarddose TMP-SMX groups, and the cumulative discontinuation rate due to adverse events was significantly lower in the low-dose group compared to that in the standarddose group. We believe that our findings can assist medical and health care professionals in determining TMPSMX dosage when considering PCP prophylaxis for patients undergoing hemodialysis.

\section{Abbreviations}

PCP: Pneumocystis pneumonia; HIV: Human immunodeficiency virus; TMPSMX: Trimethoprim-sulfamethoxazole; SS: Single strength; AE: Adverse event; CTCAE: Common Terminology Criteria for Adverse Events; IQR: Interquartile range; $\mathrm{Cl}$ : Confidence interval; aHR: Adjusted hazard ratio

\section{Acknowledgements}

The authors thank all the physicians and medical staff working for their institution.

\section{Authors' contributions}

KY designed the study and collected and analyzed the data. KY and YS performed the statistical analysis and wrote the first draft of the manuscript. $\mathrm{HI}, \mathrm{NM}, \mathrm{AY}$, and $\mathrm{TH}$ critically reviewed the analyzed data and manuscript. All authors approved the final version of the manuscript.

\section{Funding}

The authors declare no competing financial and non-financial interests.

\section{Availability of data and materials}

The datasets used and/or analyzed during the current study are available from the corresponding author on reasonable request.

\section{Declarations}

\section{Ethics approval and consent to participate}

The study was conducted in accordance with the principles of the Declaration of Helsinki. This study was approved by the ethics committee in
Kobe City Medical Center General Hospital, and the reference number was zn190616. Informed Consent was waived by the ethics committee in Kobe City Medical Center General Hospital because this study used retrospective data obtained from hospital records and there were no interventions in the study patients.

\section{Consent for publication}

Not applicable.

\section{Competing interests}

The authors declare that they have no competing interests.

\section{Author details}

${ }^{1}$ Department of Pharmacy, Kobe City Medical Center General Hospital, 2-1-1 Minatojima-minamimachi, Chuo-ku, Kobe, Hyogo 650-0047, Japan.

${ }^{2}$ Department of Hematology, Kobe City Medical Center General Hospital, Kobe, Japan. ${ }^{3}$ Department of Nephrology, Kobe City Medical Center General Hospital, Kobe, Japan.

Received: 3 April 2021 Accepted: 21 June 2021

Published online: 08 July 2021

\section{References}

1. Kaplan JE, Benson C, Holmes KK, Brooks JT, Pau A, Masur H, et al. Guidelines for prevention and treatment of opportunistic infections in HIV-infected adults and adolescents: recommendations from CDC, the National Institutes of Health, and the HIV Medicine Association of the Infectious Diseases Society of America. MMWR Recomm Rep. 2009;58 RR-4:1-207 quiz CE1-4.

2. Yale SH, Limper AH. Pneumocystis carinii pneumonia in patients without acquired immunodeficiency syndrome: associated illnesses and prior corticosteroid therapy. Mayo Clin Proc. 1996;71(1):5-13. https://doi.org/10.4 065/71.1.5.

3. Mansharamani NG, Garland R, Delaney D, Koziel H. Management and outcome patterns for adult Pneumocystis carinii pneumonia, 1985 to 1995 : comparison of HIV-associated cases to other immunocompromised states. Chest. 2000;118(3):704-11. https://doi.org/10.1378/chest.118.3.704.

4. Sepkowitz KA. Opportunistic infections in patients with and patients without acquired immunodeficiency syndrome. Clin Infect Dis. 2002;34(8): 1098-107. https://doi.org/10.1086/339548.

5. Ward MM, Donald F. Pneumocystis carinii pneumonia in patients with connective tissue diseases: the role of hospital experience in diagnosis and mortality. Arthritis Rheum. 1999;42(4):780-9. https://doi.org/10.1002/15290131(199904)42:4<780::AID-ANR23>3.0.CO;2-M.

6. Cooley L, Dendle C, Wolf J, Teh BW, Chen SC, Boutlis C, et al. Consensus guidelines for diagnosis, prophylaxis and management of Pneumocystis 
jirovecii pneumonia in patients with haematological and solid malignancies, 2014. Intern Med J. 2014;44(12b):1350-63. https://doi.org/10.1111/imj.12599.

7. Martin SI, Fishman JA, AST Infectious Diseases Community of Practice.

Pneumocystis pneumonia in solid organ transplantation. Am J Transplant. 2013;13 Suppl 4:272-9.

8. Taplitz RA, Kennedy EB, Bow EJ, Crews J, Gleason C, Hawley DK, et al. Antimicrobial prophylaxis for adult patients with cancer-related immunosuppression: ASCO and IDSA clinical practice guideline update. JCO. 2018;36(30):3043-54. https://doi.org/10.1200/JCO.18.00374.

9. Tomblyn M, Chiller T, Einsele H, Gress R, Sepkowitz K, Storek J, et al. Guidelines for preventing infectious complications among hematopoietic cell transplantation recipients: a global perspective. Biol Blood Marrow Transplant. 2009;15(10):1143-238. https://doi.org/10.1016/j.bbmt.2009.06.019.

10. DailyMed - SULFAMETHOXAZOLE AND TRIMETHOPRIM DOUBLE STRENGTHsulfamethoxazole and trimethoprim tablet. https://dailymed.nlm.nih.gov/da ilymed/druglnfo.cfm?setid=804f8363-8ea1-4a57-bbd1-7fd9b6b4d29b. Accessed 18 Dec 2020.

11. Common Terminology Criteria for Adverse Events (CTCAE). version 5.0. 2017; 155. https:/ctep.cancer.gov/protocolDevelopment/electronic_applications/ ctc.htm\#ctc_50.

12. Kanda Y. Investigation of the freely available easy-to-use software "EZR" for medical statistics. Bone Marrow Transplant. 2013;48(3):452-8. https://doi. org/10.1038/bmt.2012.244.

13. Utsunomiya M, Dobashi H, Odani T, Saito K, Yokogawa N, Nagasaka K, et al. Optimal regimens of sulfamethoxazole-trimethoprim for chemoprophylaxis of Pneumocystis pneumonia in patients with systemic rheumatic diseases: results from a non-blinded, randomized controlled trial. Arthritis Res Ther. 2017;19(1):7. https://doi.org/10.1186/s13075-016-1206-8.

14. Butler-Laporte G, Smyth E, Amar-Zifkin A, Cheng MP, McDonald EG, Lee TC. Low-dose TMP-SMX in the treatment of Pneumocystis jirovecii pneumonia: a systematic review and meta-analysis. Open Forum Infect Dis. 2020;7:ofaa112.

15. Stern A, Green H, Paul M, Vidal L, Leibovici L. Prophylaxis for Pneumocystis pneumonia (PCP) in non-HIV immunocompromised patients. Cochrane Database Syst Rev. 2014;2014:CD005590. https://doi.org/10.1002/14651858. CD005590.pub3.

16. Utsunomiya M, Dobashi H, Odani T, Saito K, Yokogawa N, Nagasaka K, et al. An open-label, randomized controlled trial of sulfamethoxazoletrimethoprim for Pneumocystis prophylaxis: results of 52-week follow-up. Rheumatol Adv Prac. 2020;4(2). https://doi.org/10.1093/rap/rkaa029.

17. Zmarlicka M, Martin ST, Cardwell SM, Nailor MD. Tolerability of low-dose sulfamethoxazole/trimethoprim for Pneumocystis jirovecii pneumonia prophylaxis in kidney transplant recipients. Prog Transplant. 2015;25(3):2106. https://doi.org/10.7182/pit2015153.

\section{Publisher's Note}

Springer Nature remains neutral with regard to jurisdictional claims in published maps and institutional affiliations.

Ready to submit your research? Choose BMC and benefit from:

- fast, convenient online submission

- thorough peer review by experienced researchers in your field

- rapid publication on acceptance

- support for research data, including large and complex data types

- gold Open Access which fosters wider collaboration and increased citations

- maximum visibility for your research: over $100 \mathrm{M}$ website views per year

At $\mathrm{BMC}$, research is always in progress.

Learn more biomedcentral.com/submissions 